\title{
Visualizing laser-skin interaction in vivo by multiphoton microscopy
}

\author{
Tsung-Hua Tsai* \\ Far Eastern Memorial Hospital \\ Department of Dermatology \\ 21, Nan-Ya South Road, Section 2 \\ Pan-Chiao, Taipei 220 \\ Taiwan \\ and \\ Oriental Institute of Technology \\ General Education Center \\ 58, Section 2, Sihchuan Road \\ Ban-Chiao City, Taipei County 220 \\ Taiwan
}

\author{
Shiou-Hwa Jee* \\ National Taiwan University Hospital \\ and College of Medicine \\ Department of Dermatology \\ 1, Section 1, Jen-Ai Road \\ Taipei 100
}

Taiwan

\section{Jung-Yi Chan}

Cathay General Hospital

Department of Dermatology

280, Section 4, Jen-Ai Road

Taipei 106

Taiwan

\section{Jin-Ning Lee}

National Taiwan University

Department of Physics

Number 1, Section 4, Roosevelt Road

Taipei 106

Taiwan

\section{Woan-Ruoh Lee}

Taipei Medical University

Graduate Institute of Medical Sciences

250, Wu-Xin Street

Taipei 100

Taiwan

\section{Chen-Yuan Dong}

National Taiwan University

Department of Physics

and Center for Quantum Science and Engineering

Number 1, Section 4, Roosevelt Road

Taipei 106

Taiwan

*Authors contributed equally to this work.

Address all correspondence to: Chen-Yuan Dong, PhD, Associate Professor, Department of Physics National Taiwan University, No. 1, Sec. 4, Roosevelt Road, Taipei 106, Taiwan; Tel: 886-2-3366-5155; Fax: 886-2-3366-5244; E-mail: cydong@phys.ntu.edu.tw; and Sung-Jan Lin, MD, PhD, Assistant Professor, Institute of Biomedical Engineering, National Taiwan University, No. 1, Sec. 1, Jen-Ai Road, Taipei 100, Taiwan; Tel: 886-2-23123456 ext 5323; Fax: 886-22392-5351; E-mail: drsjlin@ntu.edu.tw.

\author{
Sung-Jan Lin \\ National Taiwan University \\ Institute of Biomedical Engineering \\ and \\ National Taiwan University Hospital \\ and College of Medicine \\ Department of Dermatology \\ 1, Section 1, Jen-Ai Road \\ Taipei 100 \\ Taiwan
}

\begin{abstract}
Recently, multiphoton microscopy has gained much popularity as a noninvasive imaging modality in biomedical research. We evaluate the potential of multiphoton microscopy for monitoring laser-skin reaction in vivo. Nude mouse skin is irradiated with an erbium:YAG laser at various fluences and immediately imaged by a multiphoton microscope. The alterations of cutaneous nonlinear optical properties including multiphoton autofluorescence and second-harmonic generation associated with laser irradiation are evaluated morphologically and quantitatively. Our results show that an erbium:YAG laser at a low fluence can selectively disrupt the stratum corneum, and this alteration may account for the penetration enhancing effect of laser-assisted transcutaneous drug delivery. At a higher fluence, the zone of tissue ablation as well as the disruption of the surrounding stratum corneum, keratinocytes, and dermal extracellular matrix can be better characterized by multiphoton microscopy as compared with conventional histology. Furthermore, the degree of collagen damage in the residual thermal zone can be quantified by second-harmonic generation signals, which have significant difference between control skin, skin irradiated with a $1.5-, 8-$, and $16-\mathrm{J} / \mathrm{cm}^{2}$ erbium:YAG laser $(P<0.05)$. We show that multiphoton microscopy can be a useful noninvasive imaging modality for monitoring laser-skin reaction in vivo. () 2009 Society of Photo-Optical Instrumentation Engineers. [DOI: 10.1117/1.3116711]
\end{abstract}

Keywords: multiphoton; autofluorescence; second-harmonic generation; laser; laser-assisted drug delivery; collagen.

Paper 08296R received Aug. 27, 2008; revised manuscript received Dec. 4, 2008; accepted for publication Feb. 11, 2009; published online Apr. 22, 2009.

\section{Introduction}

Various light therapies have been employed in clinical practice. Advances in these treatment modalities, such as various types of laser, intense pulsed light (IPL), and light emitting diodes, have led to their wide clinical applications in treating cutaneous diseases and performing cosmetic procedures. ${ }^{1}$

The results of laser treatment depend on the immediate interaction of incident light with tissues well as the subsequent tissue regeneration and remodeling. As in many areas of medicine, histological examinations are necessary to evaluate

$1083-3668 / 2009 / 14(2) / 024034 / 8 / \$ 25.00$ ○ 2009 SPIE 
the reaction of epidermal, dermal, vascular, and appendageal components of skin after laser therapy. ${ }^{2}$ The major drawback of conventional histological examination is its invasive nature and the inability to examine the light-tissue interaction in real time. The use of inappropriate parameters can result in inadequate treatment outcome and may lead to serious side effects. ${ }^{3}$ To be specific, skin conditions can vary among individuals as well as among the treated areas in the same individual. To achieve a favorable result, the parameters employed should be adjusted accordingly. Despite the wide application of light therapy, the association between immediate light-tissue reaction, subsequent tissue remodeling, and final clinical outcome has not been investigated in detail due to the lack of an appropriate real-time noninvasive monitoring tool. As a result, validation of appropriate adjustable clinical treatment parameters often relies on repetition of trial-anderror practice.

Investigators have resorted to noninvasive objective methods to assess laser-tissue reaction, such as sonography and optical coherence tomography. ${ }^{4,5}$ However, limited by the resolution, these techniques do not enable detailed analysis of cutaneous structural alterations. Recently, reflectant confocal microscopy has gained popularity as a useful clinical tool for noninvasive imaging by providing subcellular resolution. ${ }^{6}$ Though it is powerful in analyzing the cellular morphologies in epidermis, its ability to resolve the turbid dermal extracellular matrical structures is limited.

Recently, the nonlinear optical imaging technique of multiphoton microscopy (MPM) has emerged as a useful technique for skin imaging. ${ }^{7-10}$ In MPM, fluorophores are excited by simultaneous absorption of two or more photons in the near-IR spectrum as opposed to absorption of one photon in the UV or visible light spectrum in conventional florescence microscopy. To achieve efficient multiphoton excitation, extremely high peak photon input from lasers with pulses in the range of tens to hundreds of femtoseconds is required. ${ }^{7}$ Due to the nonlinearity, the excitation occurs only at the focal point and images from a thin optical section can be acquired without using a pinhole. MPM holds several unique advantages over confocal scanning laser microscopy, such as reduced specimen photobleaching, higher contrast images, and enhanced penetration depth. ${ }^{7,9,11}$ Since endogenous autofluorescent substances including $\mathrm{NAD}(\mathrm{P}) \mathrm{H}$, keratin, and elastic fibers can be excited by multiphoton excitation, label-free imaging can be achieved. ${ }^{9,11}$

By use of a femtosecond laser, the nonlinear polarization effect of second-harmonic generation (SHG) can also be used for visualizing biological structures lacking inversion symmetry. ${ }^{11,12}$ For SHG, the interaction of noncentrosymmetric structures with incident light yields photons with a wavelength of exactly half the wavelength of the incident light. Unlike fluorescence excitation, the polarization effect of SHG does not involve absorption of the incident photons by the molecules. Therefore, photobleaching and thermal damage can be avoided. Collagen fibers, myosin, and microtubules are effective biological SHG signal generators. ${ }^{12}$ As SHG is highly sensitive to structures, SHG signals can be used for probing modification of collagen structures in cancer and evaluating the thermal structural transitions of collagen. ${ }^{13,14}$ The wavelength of SHG is shorter than the fluorescence generated by multiphoton excitation, and hence, SHG signals can be easily separated from fluorescence for further analysis and image processing. The combination of multiphoton autofluorescence (AF) and SHG imaging has been applied to evaluating cutaneous aging and differentiating basal cell carcinoma from normal dermal stroma. ${ }^{15,16}$ Multiphoton microscopy has been used to detect corneal architecture and intratissue femtosecond laser effect in a rabbit eye model. ${ }^{17}$

In this paper, we assessed the usefulness of MPM in monitoring laser-skin reactions in vivo. An erbium:YAG (Er:YAG) laser was chosen in this study due to its wide dermatological applications, such as dermabrasion, skin tumor removal, skin rejuvenation, and enhancement of transcutaneous drug delivery. ${ }^{18,19}$ We investigated the morphological and quantitative changes of AF and SHG signals associated with Er:YAG laser irradiation and evaluated the potential of MPM in realtime evaluation of laser-skin interaction.

\section{Materials and Methods}

\subsection{Multiphoton Microscope}

The multiphoton microscope is similar to the one we previously described with modification. ${ }^{20}$ A femtosecond titaniumsapphire (ti-sa) laser (Tsunami; Spectra Physics, Mountain View, California) pumped by a diode-pumped solid state (DPSS) laser (Millennia X; Spectra Physics, Mountain View, California) was used as the excitation source. The 780-nm output of the laser system was guided toward a modified commercial upright microscope (E800; Nikon, Tokyo, Japan). Prior to entering the microscope, the excitation source was angularly deflected by an $x$ - $y$ scanning system (Model 6220; Cambridge Technology, Cambridge, Massachusetts). The input of the upright microscope was modified to accommodate a beam expander. The excitation source was beam expanded and reflected toward the focusing objective [S Fluor $40 \times$, numerical aperture $(\mathrm{NA})=1.30$; Nikon, Tokyo, Japan] by a short-pass primary dichroic (700DCSPXRUV-3p; Chroma Technology, Rockingham, Vermont). The power at the sample was $35 \mathrm{~mW}$. The nonlinear AF and SHG signals were generated at the sample focal plane and collected in the epiilluminated or back-scattering geometry by the same focusing objective. After passing through the primary dichroic, the AF and SHG signals were separated into two separate channels where they were detected by independent photomultiplier tubes (R7400P; Hamamatsu, Hamamatsu City, Japan). The AF and SHG signals were separated by a secondary dichroic (435DCXR; Chroma Technology Rockingham, Vermont). The secondary dichroic is a long-pass filter that reflects below and transmits above $435 \mathrm{~nm}$. The SHG signal was further filtered by a SHG filter (HQ390/20; Chroma Technology, Rockingham, Vermont) centered at $390 \mathrm{~nm}$ with a bandwidth of $20 \mathrm{~nm}$, while the AF was detected by a bandpass filter (E700sp-2P-E435lp; ChromaTechnology, Rockingham, Vermont) for broadband fluorescence detection between 435 and $700 \mathrm{~nm}$. To acquire a global image of the thermally treated specimens at high resolution, an $x-y$ sample positioning stage (H101; Prior Scientific Instruments, Cambridge, UK) was used to translate the skin specimen after each imaged frame. An array of overlapping and individually beam-scanned images, each with an area of $110 \times 110 \mu \mathrm{m}^{2}$, was then assembled. 

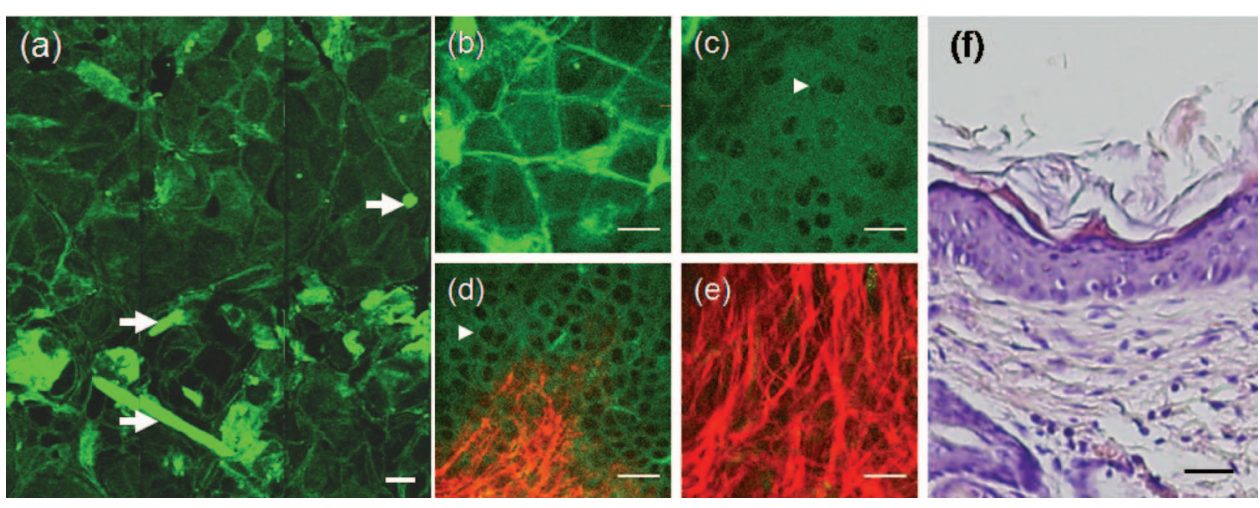

Fig. 1 MPM images and histology of unirradiated nude mouse skin (a) to (e) MPM images. The green channel represents AF and the red channel represents SHG. (a) MPM image of the skin surface. The stratum corneum is autofluorescent and the morphology of corneocytes can be clearly demonstrated. Hair is also intensely autofluorescent (arrows). (b) Magnified picture of the skin surface. (c) MPM image of the stratum spinosum, $32 \mu \mathrm{m}$ from the surface. Keratinocytes with abundant autofluorescent cytoplasm surrounding round nonfluorescent nuclei (arrowhead) can be demonstrated. (d) Basal layer and the dermal-epidermal junction, $80 \mu \mathrm{m}$ from the skin surface. Basal keratinocytes (arrowhead) are smaller with scanty cytoplasm as compared with that in stratum spinosum. SHG emitted from fibrous collagen starts to appear in the dermal-epidermal junction. (e) MPM image of the dermis, $160 \mu \mathrm{m}$ from skin surface. Fibrous collagen bundles with intense SHG signals are penetrating in the dermis. (f) The corresponding histological image. Compared with MPM images, the stratum corneum is loose with a basket-weave pattern due to artifacts from the fixation and staining process. (Bars: $20 \mu \mathrm{m}$.)

\subsection{Image Processing and Analysis}

The MPM images were analyzed and reconstructed by the software ImageJ 1.36b (National Institutes of Health, Bethesda, Maryland). The toolbox enabled visual inspection of various morphologic features and fluorescence patterns of cellular components within normal skin and laser-irradiated skin. AF and SHG signals of each site were quantitatively acquired. To determine whether treatment with Er:YAG laser at various fluences influences dermal SHG, average SHG intensity at a depth of more than $60 \mu \mathrm{m}$ of untreated and treated skin were analyzed with a general linear model. A value of $P=0.05$, corresponding to the $95 \%$ confidence interval, was selected for analysis of significant difference of SHG in this study. SPSS 15.0 statistical software (SPSS Inc., Chicago, Illinois) was used.

\subsection{Er:YAG Laser Assembly}

An Er:YAG laser (Burane; WaveLight Laser Technologie AG, Erlangen, Germany) was used to irradiate skin. The laser had a wavelength of $2940 \mathrm{~nm}$ and pulse duration of $250 \mu \mathrm{s}$. An articulated arm was used to deliver the laser beam onto the skin. Laser beam spot size was $1.5 \mathrm{~mm}$ in diameter. Each skin site was exposed once.

\subsection{Accommodation of the Mouse on the Microscopic Stage}

The experimental protocol involving animals was approved by our Institutional Animal Care and Use Committee. Male nude mice (Balb/c-nu strain, 8 to 10 weeks old) were anesthetized by intraperitoneal phenobarbitol injection and prepared for Er:YAG laser irradiation. Immediately after laser irradiation, mouse dorsal skin were stabilized in a dorsal skin fold chamber, coverslipped, and observed under the MPM.

\subsection{Histological Examination of Skin}

Full-thickness dorsal skin after MPM imaging was excised. The skin specimens were fixed in $10 \%$ buffered formalin so- lution and further processed for histological examinations with hematoxylin and eosin stains. The adjacent normal skin was assessed as the control.

\section{Results}

\subsection{Multiphoton Image of Unirradiated Nude Mouse Skin}

Figure 1 shows the multiphoton images of unirradiated nude mouse skin. MPM images of skin surface reveal compact anucleated corneocytes. Autofluorescent pentagonal or hexagonal corneocytes are tightly packed and the cell borders are clearly delineated [Figs. 1(a) and 1(b)]. Hair is also intensely autofluorescent and can be seen on the skin surface (arrows). In the optical sections corresponding to the stratum spinosum and stratum basale, the cells are characterized by fluorescent cellular cytoplasm surrounding nonfluorescent round nuclei. A gradual decrease in cell size can be observed when serial images are taken from the stratum spinosum down to the stratum basale. The ratio of the nucleus to the cytoplasm also increases with depth [Figs. 1(c) and 1(d)]. The dermalepidermal junction with intermingled AF signals from basal keratinocytes and SHG signals from collagen fibers can be seen in Fig. 1(d). Collagen fibers in dermis emit strong SHG signals [Fig. 1(e)].

In the corresponding histological image, in contrast to compact structures revealed in the MPM images, stratum corneum is loose with a basket-weave pattern [Fig. 1(f)]. This discrepancy is due to the artifacts caused by fixation and staining procedures in histological examinations that disrupt the normal intercellular packing of corneocytes. ${ }^{21-23}$ In addition, the epidermis is thinner measured histologically (about $60 \mu \mathrm{m})$ than that measured by multiphoton microscopy (about $80 \mu \mathrm{m}$ ). This indicates that tissue shrinkage during processing of the specimen for histological examination can be a significant artifact that interferes with the precise measurement of tissue thickness. 

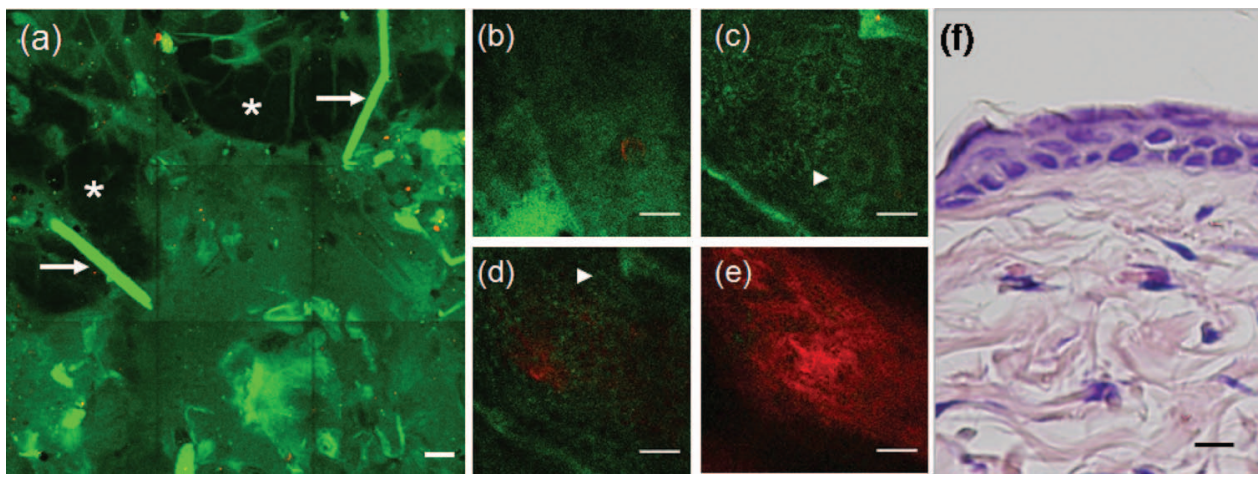

Fig. 2 MPM images and histology of nude mouse skin irradiated with Er:YAG laser at the fluence of $1.5 \mathrm{~J} / \mathrm{cm}^{2}$. (a) to (e) MPM images. The green channel represents AF and the red channel represents SHG. (a) MPM image of the skin surface. The fluorescent stratum corneum becomes a homogenized morphology. The focal stratum corneum is loosened or absent (asteroids). Brightly fluorescent hairs are still intact (arrows). (b) A magnified picture of skin surface. (c) MPM image of stratum spinosum, $30 \mu \mathrm{m}$ from skin surface. Intact keratinocytes with fluorescent cytoplasm can still be observed (arrowhead). (d) Basal layer and the dermal-epidermal junction, $70 \mu \mathrm{m}$ from skin surface. Smaller fluorescent basal cells (arrowhead) and SHG-emitting collagen can be seen. (e) MPM image of dermis, $130 \mu \mathrm{m}$ from skin surface. The fibrous structures of collagen can still be observed but the detected SHG intensity is decreased compared with unirradiated skin. (f) Corresponding histological image. Compared with the MPM image, the stratum corneum is almost totally detached during fixation and staining process. Structural alterations can be hardly seen in the underlying structures. (Bars: $20 \mu \mathrm{m}$.)

\subsection{Selective Disruption of Stratum Corneum by Er:YAG Laser at a Low Fluence of $1.5 \mathrm{~J} / \mathrm{cm}^{2}$ Revealed by Multiphoton Imaging}

Nude mouse skin was irradiated with Er:YAG laser at a fluence of $1.5 \mathrm{~J} / \mathrm{cm}^{2}$. In the MPM images, compared with the tightly packed hexagonal corneocytes with distinctive intercellular borders in control group [Fig. 1(a)], the morphology of a large proportion of corneocytes has been disrupted into a homogenized pattern and intercellular borders can not be clearly delineated [Figs. 2(a) and 2(b)]. In addition to the homogenization change, selective loosening of stratum corneum and loss of corneocytes can also be observed focally [Fig. 2(a), asteroids]. Brightly fluorescent hairs are still intact and are relatively resistant to laser irradiation (arrows). However, in the corresponding histological image, only scanty corneocytes are still preserved [Fig. 2(f)]. We speculate that the homogenized or loosened corneocytes are lost during histological processing.

When images are taken farther down the skin surface, the morphology of keratinocytes in the stratum spinosum and stratum basale is still intact [Figs. 2(c) and 2(d)]. The SHG signals can still be detected from the dermal collagen [Fig. 2(e)]. In the histological image, epidermal keratinocytes are also still intact and no significant change of dermal collagen is revealed [Fig. 2(f)]. Since the AF from the epidermal cells and SHG from the dermis are all decreased and the cellular morphology and collagen fibers are relatively intact, the decrease of the intensity of AF and SHG from epidermal cells and dermal collagen may be caused by the shielding effect from the intensely fluorescent disrupted corneocytes.

\subsection{Ablation of Stratum Corneum and Thermal Injury to Underlying Structures by Er:YAG Laser at Moderate Fluence of $8 \mathrm{~J} / \mathrm{cm}^{2}$}

After irradiation at the fluence of $8 \mathrm{~J} / \mathrm{cm}^{2}$, partial ablation of stratum corneum is revealed and the residual stratum corneum displays a loose reticular pattern [Figs. 3(a) and 3(b)]. The stratum spinosum starts to become a homogenous fluorescent morphology without discernable intercellular borders [Fig. 3(c)]. Residual discernable keratinocytes are mixed with homogenized cells in basal layer [Fig. 3(d)]. In the corresponding histological image, intact corneocytes can hardly be seen. There is a thin layer of eosinophilic debris in which upper epidermal cells show a condensed nucleus and more eosinophilic cytoplasm. Some of these cells are partially detached from the underlying epidermal cells. Many residual basal cells become vacuolated [Fig. 3(f), arrowhead].

When the images are taken farther down to the dermis, the intensity of SHG signals from the dermis is greatly diminished and the fibrous morphology of collagen is further deteriorated into an amorphous structure due to the residual thermal damage from laser irradiation [Fig. 3(e)]. Compared with MPM images, this denatured collagen zone can not be easily appreciated in the histology. It presents as a thin layer of slightly homogenized collagen immediately beneath dermalepidermal junction [Fig. 3(f)].

\subsection{Complete Ablation of Epidermis with a Thermal Injury Zone to the Dermis by Er:YAG Laser at a High Fluence of $16 \mathrm{~J} / \mathrm{cm}^{2}$}

High-fluence laser irradiation $\left(16 \mathrm{~J} / \mathrm{cm}^{2}\right)$ produced a depressed crateriform hole [Fig. 4(a)]. On the rim of the crateriform hole, the residual corneocytes and keratinocytes are homogenized into a highly fluorescent ring [Fig. 4(a)]. In the central area of the crateriform hole, the stratum corneum can not be seen after being ablated. When images are taken down to the skin in the central area [Figs. 4(b)-4(e)], intact keratinocytes can not be observed. In the dermis, no SHG signals can be detected [Fig. 4(e)]. In the histological image, no residual epidermal cells are observed [Fig. 4], and the crater can not be easily appreciated due to tissue shrinkage and deformation. Eosinophilic homogenized degeneration of dermal collagen can be seen in the central area of the ablated zone [boxed area, Fig. 4(f)]. 

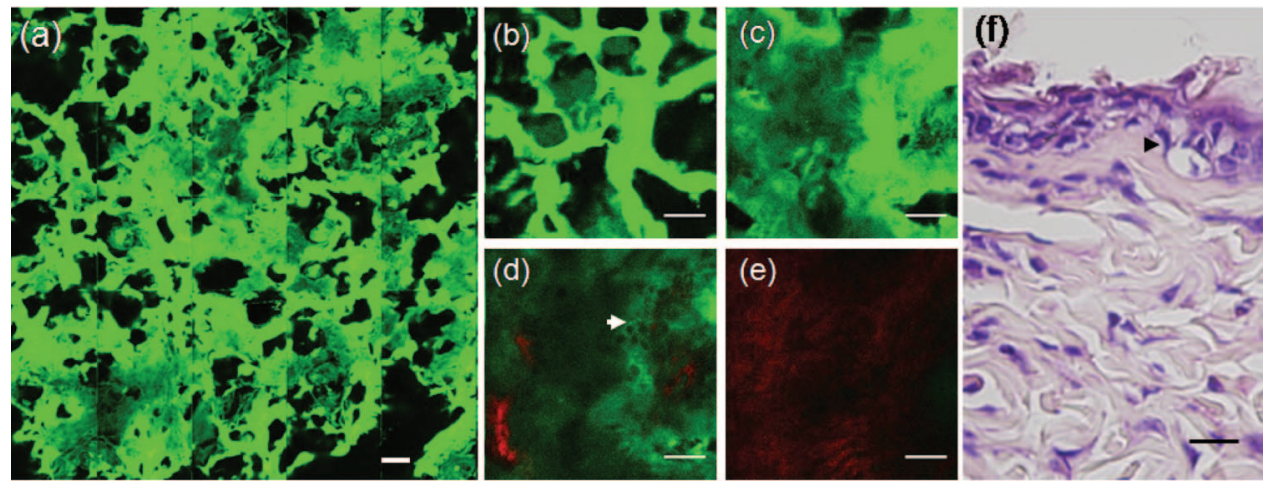

Fig. 3 MPM images and histology of nude mouse skin irradiated with Er:YAG laser at the fluence of $8 \mathrm{~J} / \mathrm{cm}^{2}$. (a) to (e) MPM images. The green channel represents AF and the red channel represents SHG. (a) MPM image of skin surface. Intensely fluorescent residual stratum corneum is intermingled with focal ablated nonfluorescent areas. Hairs disappear due to vaporization at this fluence. (b) Magnified picture of skin surface. (c) MPM image of stratum spinosum, $40 \mu \mathrm{m}$ from skin surface. Irradiated stratum spinosum also becomes homogenized without distinctive cell borders. (d) The basal layer and the dermal-epidermal junction, $80 \mu \mathrm{m}$ from skin surface. Homogenized keratinocytes are mixed with a few discernible keratinocytes (arrow). (e) MPM image of dermis, $180 \mu \mathrm{m}$ from skin surface. SHG signals from collagen are greatly diminished and the fibrous morphology of collagen is also disrupted from thermal denaturation. (f) Corresponding histological image. A thin layer of eosinophilic residual debris is observed. Basal keratinocytes have a ballooning and vacuolated morphology (arrowhead). The collagen in the papillary dermis is slightly homogenized. (Bar: $20 \mu \mathrm{m}$.)

\subsection{Probing the Laser-Induced 3-D Structural Alterations by Multiphoton Imaging}

To highlight the spatial impact of laser irradiation on skin, the serial multiphoton images are reconstructed into 3-D images (Fig. 5). In unirradiated skin, keratin and keratinocytes form an autofluorescent layer from the top of the image and fibrous collagen emitting SHG signals are penetrating in the dermis [Fig. 5(a)]. The dermal-epidermal junction shows an undulating plane between the AF and SHG signals. The crater generated by high-fluence Er:YAG laser irradiation $\left(16 \mathrm{~J} / \mathrm{cm}^{2}\right)$ can be more easily visualized in the reconstructed image [Fig. $5(\mathrm{~b})]$. The shape of the crater is consistent with the reverse bell-shaped Gaussian distribution of the laser beam. The absence of SHG signal in residual dermis indicates that the collagen is denatured in the residual thermal zone.

\subsection{Quantitative SHG Analysis Detects the Thermal Injury Profile in Laser-Irradiated Skin}

It has been reported that the change in the intensity of SHG is a good predictor of collagen structural disruption. ${ }^{13,14,24}$ We quantitatively analyzed SHG intensity after laser irradiation at various fluences. Figure 6 shows the SHG intensity profiles of normal skin and skin irradiated with a laser at various fluences. In the unirradiated skin, the intensity of SHG started to increase between 40 and $80 \mu \mathrm{m}$ from the surface. This zone corresponds to the undulating dermal-epidermal junction. SHG intensity approaches its plateau at the depth of about 80 to $120 \mu \mathrm{m}$ from the surface. This corresponds to the plentiful collagen content in the dermis. After irradiated with Er:YAG laser at low fluence of $1.5 \mathrm{~J} / \mathrm{cm}^{2}$, the plateau inten-
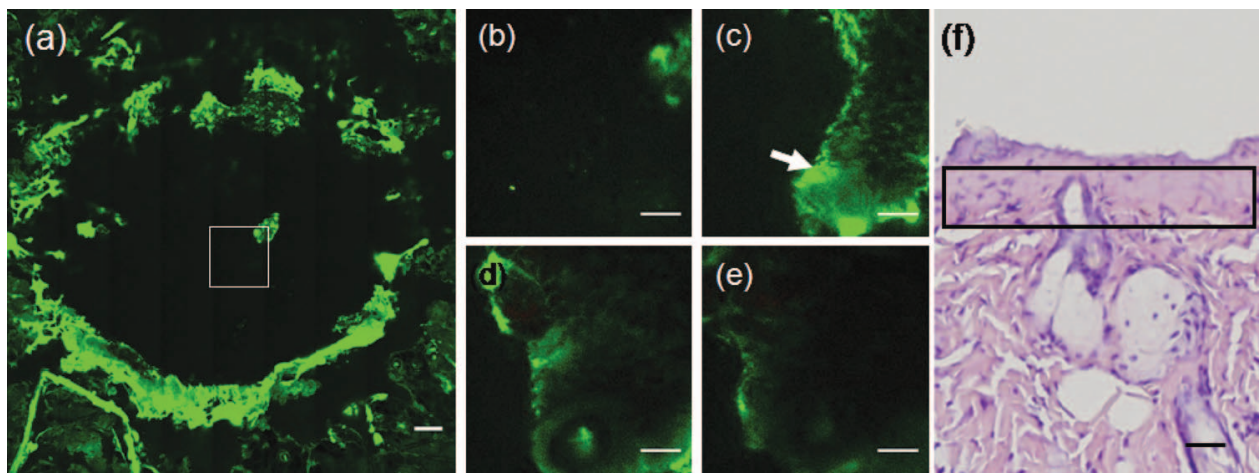

Fig. 4 MPM images and histology of nude mouse skin irradiated with Er:YAG laser at the fluence of $16 \mathrm{~J} / \mathrm{cm}^{2}$. (a) to (e) MPM images. The green channel represents AF and the red channel represents SHG. (a) MPM image of skin surface. An ablated hole is surrounded by intensely fluorescent debris. Serial images from the boxed area along the $z$ axis are shown in (b) to (e). (b) Magnified surface of at the central hole. The space is empty due to ablation. (c) At $40 \mu \mathrm{m}$ from skin surface, residual fluorescent debris (arrow) can sometimes be seen in the ablated crater. (d) At $80 \mu \mathrm{m}$ from skin surface, only residual amorphous debris is observed and intact cells are absent. (e) At $180 \mu \mathrm{m}$ from skin surface, SHG is absent and replaced by some fluorescent materials. (f) Corresponding histological image. Eosinophilic homogenization of collagen is observed in residual thermal zone (boxed area) as compared with the normal fibrous structures of collagen beneath. [Bar: $50 \mu \mathrm{m}$ in (a); $20 \mu \mathrm{m}$ in (b) to (f).] 


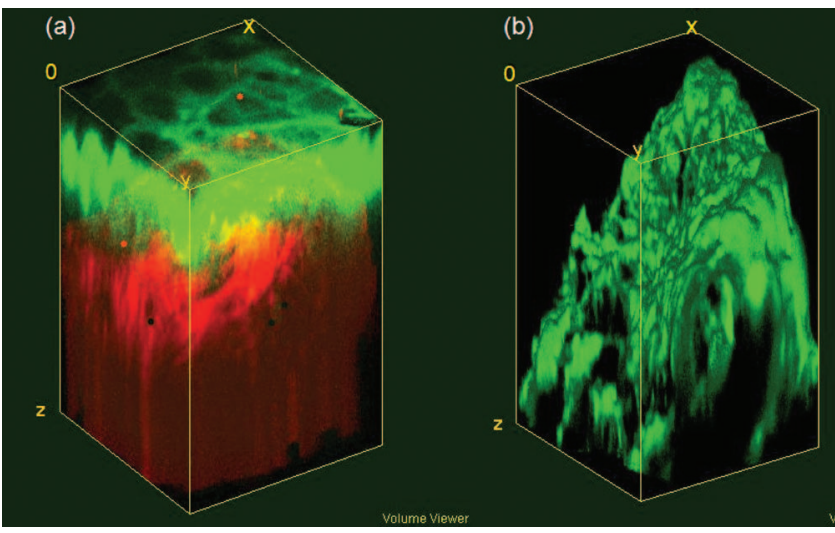

Fig. 5 Three-dimensional MPM images of control and laser irradiated skin. The green channel represents $\mathrm{AF}$ and the red channel represents SHG. (a) Normal skin has keratinocytes that emit AF in the epidermal layer and fibrous collagen that emits SHG in the dermal layer. Hexagonal tightly packed corneocytes can be seen from the top view. The dermal-epidermal junction can be delineated by the undulating waveshaped junction of AF and SHG. (b) After irradiation with Er:YAG laser at fluence of $16 \mathrm{~J} / \mathrm{cm}^{2}$, a slope of hole appears. The gradually depressed shape of the crater is consistent with the reverse bell-shaped Gaussian distribution of light intensity across the laser beam. SHG signals are absent in the dermis due to thermal denaturation. $(x-y$ plane: surface of skin $110 \times 110 \mu \mathrm{m}^{2} ; z$ axis: skin depth $180 \mu \mathrm{m}$.)

sity of SHG signals in the dermis decreases compared with the control group. However, the increase of SHG intensity at the dermal-epidermal junction of depth about 40 to $80 \mu \mathrm{m}$ is still observed. Since the fibrous structures of collagen are still observed, the decrease in the plateau SHG intensity in dermis may be caused by the shielding effect of the overlying homogenized highly fluorescent stratum corneum. At the moderate fluence of $8 \mathrm{~J} / \mathrm{cm}^{2}$, SHG decreases more significantly and the increasing trend of intensity at the dermal-epidermal junction can only be barely observed. In skin exposed to a high fluence of $16 \mathrm{~J} / \mathrm{cm}^{2}$, the SHG signals are undetectable. SHG signals of dermal collagen are significantly different between four groups with paired comparisons $(P<0.05)$.

\section{Discussion}

The Er:YAG laser and the 10,600-nm $\mathrm{CO}_{2}$ laser are two commonly used resurfacing lasers. The epidermis is composed of $80 \%$ water. In these two lasers, the major chromophore for absorbance is water. At high fluences of the $\mathrm{CO}_{2}$ laser for ablation, instant heating of water to $100{ }^{\circ} \mathrm{C}$ occurs and vaporization of tissue ensues. However, it can also yields a zone of coagulation beyond the vaporization zone to a variable degree, depending on the mode and parameters of $\mathrm{CO}_{2}$ laser used. ${ }^{25}$ The residual thermal effects in the temperature range of 40 to $100{ }^{\circ} \mathrm{C}$ can result in denaturation of proteins, unwinding of DNA, and disruption of cell membranes. Though the residual thermal zone of the $\mathrm{CO}_{2}$ laser can contribute to tissue shrinkage for the purpose of tightening in skin rejuvenation, it can also lead to undesirable effects. For example, this residual thermal effect can compromise cell function and necrosis ensues. ${ }^{26}$

With the Er:YAG laser at $2940 \mathrm{~nm}$, the energy is so efficiently absorbed by water (12 to 18 times that of the

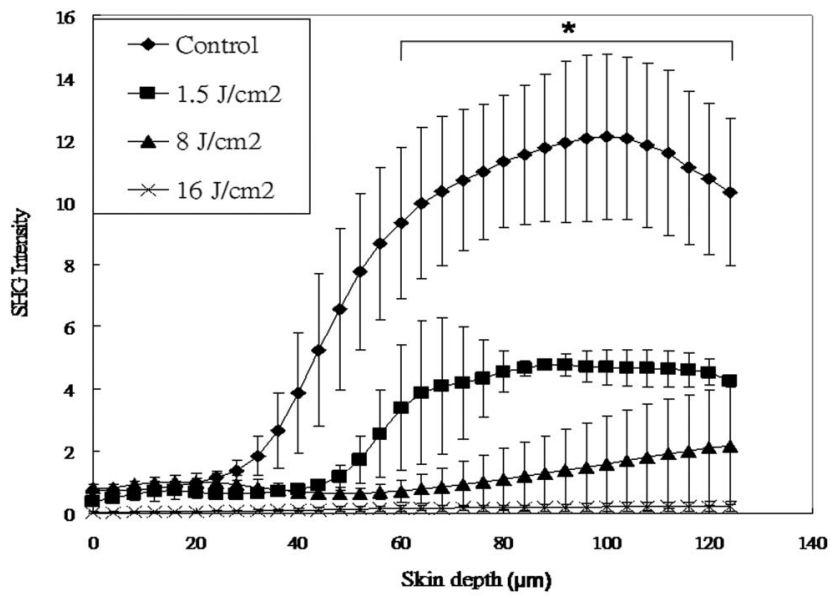

Fig. 6 SHG intensity profiles of skin irradiated with Er:YAG laser at various fluences as a function of skin depth $(z)$ from the skin surface (at $z=0 \mu \mathrm{m}$ ). Unirradiated control skin has higher SHG along the depth since collagen appears in the dermal-epidermal junction at the depth of about 40 to $80 \mu \mathrm{m}$. SHG intensity rapidly increases to a plateau value at the depth of 80 to $120 \mu \mathrm{m}$ from the surface. After Er:YAG laser irradiation at a low fluence of $1.5 \mathrm{~J} / \mathrm{cm}^{2}$, the plateau intensity of the SHG signals in the dermis decreases compared with the control group. At a moderate fluence of $8 \mathrm{~J} / \mathrm{cm}^{2}$, the SHG signal further decreases and the increasing trend at the depth of 40 to $80 \mu \mathrm{m}$ is also greatly diminished. At a high fluence of $16 \mathrm{~J} / \mathrm{cm}^{2}$, the SHG signals decrease to an undetectable level. Error bars represent $1 \mathrm{SD}$ (standard deviation) in the average value of five skin samples. Single asterisks $(*)$ indicate regions of significant difference $(P<0.05)$ between each group.

$10,600 \mathrm{~nm}$ wavelength) that its shorter penetration depth results in a more precise zone of tissue ablation with reduced residual thermal injury compared with $\mathrm{CO}_{2}$ laser. ${ }^{27}$ The Er:YAG laser is currently widely used in dermatology as an ablative tool for skin tumor removal, acne scar treatment, and facial rejuvenation. The vaporizing effect of Er:YAG laser ablates superficial tissue, whereas the residual heat conduction can still lead to tissue coagulation and collagen denaturation. ${ }^{2}$ Though the residual thermal zone can induce collagen shrinkage and neogenesis, ${ }^{28}$ too much thermal damage can also lead to unwanted complications, including postinflammatory hyperpigmentation and scars. ${ }^{3}$ The evaluation of residual thermal zones previously depended on histological examinations, but the procedure does not allow for real-time imaging. When the collagen is totally coagulated, eosinophilic homogenization can be seen in histological examination. However, if collagen is not heated enough to become totally coagulated, it can be difficult to detect the subtle changes in histology. ${ }^{24,29}$ Thermal denaturation results in the loss of molecular order in the collagen fibers, which can have a profound influence on SHG. ${ }^{13,14,24}$ Consistent with our previous reports, ${ }^{24,29}$ thermal treatment can disrupt regular collagen packing and hence the SHG signal in dermis decreases. Decrease in intensity of the SHG signals from collagen, which have statistically significant difference between each group, is correlated to the fluence of the laser (Fig. 6). The findings in this study support that fact that the SHG signal in MPM is a more sensitive tool in identifying the degree and extent of residual thermal damage in laser-irradiated skin. Hence, the SHG signals may be 
used as a real-time feedback system during laser treatment to visualize and quantify the residual thermal zone.

In addition to tissue ablation by Er:YAG laser at high fluence, Er:YAG laser at low fluence can enhance the transdermal delivery of many lipophilic and hydrophilic drugs and materials, including 5-aminolevulinic acid, 5-fluorouracil, vitamin C, and even macromolecular DNA. ${ }^{19,30-32}$ Though the detailed mechanism is unknown, it is believed that stratum corneum might be disrupted or partially ablated to achieve such a penetration enhancing effect. Previously, we could only assess the stratum corneum structural alterations in histology. However, the processing for histological examination compromises the compact structures of stratum corneum and can lead to detachment of superficial corneocytes [Fig. 2(f)]. Another drawback of histology is that thin vertical sections do not allow large-area or 3-D analysis of stratum corneum structures. The advantages of MPM in imaging stratum corneum over conventional histological examinations include the abilities to image skin in vivo without processing artifact and to provide more information by en face large-area and 3-D imaging. ${ }^{33}$ In this paper, we demonstrate that the structural changes of stratum corneum after low-energy Er:YAG laser treatment can be better highlighted by MPM than histological examinations. By use of MPM, we show that Er:YAG laser can selectively disrupt the compact structure of the stratum corneum at a low fluence while little ablative effect takes place (Fig. 2). The homogenized morphology of cornecytes is similar to the structural alterations induced by a depilatory cream, which has been reported to be able to enhance the transdermal penetration of both small model drugs and insulin. ${ }^{33-35}$ This is the first report of stratum corneum structural alterations after low-fluence Er:YAG laser irradiation by use of MPM. It has been demonstrated that, in combination with appropriate model fluorescent drugs, MPM can be used to study transcutaneous drug delivery in vitro. ${ }^{33,36-38}$ Our findings suggest that MPM can be an ideal tool for studying the mechanism and dynamics of laser-assisted transdermal drug delivery in vivo.

There is a Gaussian distribution of intensity across most kinds of laser beams and this variation of irradiance across the beam can lead to variation in biological effects in the irradiated skin. The Gaussian profile has peak intensity in the center and minimal intensity at the beam edges. ${ }^{39}$ It is important to monitor the treatment effect across the laser beam to avoid over- and undertreatment in the irradiated spot. Previously, analysis of the extent and depth of tissue alterations by laser irradiation involved labor-intensive and manual-counting methods that required multiple histological tissue sections. ${ }^{40}$ In addition, the processing of skin samples for the histological examination also leads to tissue distortion and shrinkage, and it can be difficult to precisely evaluate the impact of the variation in the intensity profile across the laser beam on the laserskin interaction. In this paper, we demonstrate that a laserinduced reverse bell-shaped slope compatible with Gaussian distribution in 3-D MPM images (Fig. 5). The laser beam has a central peak irradiance and can ablate more tissue, whereas beam edges have the lowest intensity that can only produce a shallow rim or induce nonablative thermal effects. This result can not be clearly demonstrated in the histology. Hence, MPM can be used to study the biological impact of the uneven distribution of energy within a laser beam and to facili- tate the design of laser beam intensity profile to obtain a better clinical result.

In addition to ablative lasers, other lasers have been developed for specific clinical applications including treatment of vascular abnormalities, depilation, etc. Suggested treatment parameters are often based on the results from many modeling studies, which are designed to predict photothermal effect for selective photocoagulation. ${ }^{41}$ However, current modeling methods can not adequately incorporate the complex dynamical interaction between light and tissues. MPM may also be used for monitoring the biological effects of theses lasers in vivo as well. For example, with appropriate labeling, MPM enables detailed 3-D visualization of vascular structures and quantification of hemodynamics in vivo. ${ }^{42,43}$ Cutaneous blood vessels have complex 3-D arrangement that is difficult to be appreciated in standard 2-D display in histological examination. With the reconstructed 3-D images from MPM and image analysis, it will be able to reveal the detailed vascular structures and associated changes after laser treatment and also the remodeling process.

In conclusion, by use of Er:YAG laser as the irradiation source, we demonstrate that MPM is able to probe the laserskin reaction in vivo without labeling procedures. The selective disruption of stratum corneum at low fluence and the ablative effects as well as the residual thermal injury at higher fluences can be visualized and quantified with subcellular resolution. MPM has been used in imaging human epidermal and dermal structures in vivo. ${ }^{44,45}$ Our results suggest that MPM can be further developed into a real-time feedback system for evaluating human responses to laser treatment in vivo.

\section{Acknowledgments}

This study was supported by grants from Far Eastern Memorial Hospital, Taiwan (FEMH-B95-01, FEMH-96-C29, and 97-FTN15), National Taiwan University Hospital (97S-842 and 97-FTN15), and National Research Program for Genomic Medicine of the National Science Council, Taiwan (NSC-963112-B-002-025, NSC-97-3112-B-002-037). The experiments and data analysis involving multiphoton microscopy were performed at the Optical Molecular Imaging Microscopy Core Facility, National Research Program for Genomic Medicine, Taiwan.

\section{References}

1. R. R. Anderson, "Lasers in dermatology—a critical update," J. Dermatol. 27, 700-705 (2000).

2. E. V. Ross, J. R. McKinlay, F. P. Sajben, C. H. Miller, D. J. Barnette, K. J. Meehan, N. P. Chhieng, M. J. Deavers, and B. D. Zelickson, "Use of a novel erbium laser in a Yucatan minipig: a study of residual thermal damage, ablation, and wound healing as a function of pulse duration," Lasers Surg. Med. 30, 93-100 (2002).

3. E. L. Tanzi and T. S. Alster, "Side effects and complications of variable-pulsed erbium:yttrium-aluminum-garnet laser skin resurfacing: extended experience with 50 patients," Plast. Reconstr. Surg. 111, 1524-1529 (2003).

4. B. R. Moody, J. E. McCarthy, and G. J. Hruza, "Collagen remodeling after 585-nm pulsed dye laser irradiation: an ultrasonographic analysis," Dermatol. Surg. 29, 997-999 (2003).

5. B. A. Torkian, S. Guo, A. W. Jahng, L. H. Liaw, Z. Chen, and B. J. Wong, "Noninvasive measurement of ablation crater size and thermal injury after $\mathrm{CO} 2$ laser in the vocal cord with optical coherence tomography," Otolaryngol.-Head Neck Surg. 134, 86-91 (2006).

6. M. Horn, A. Gerger, S. Koller, W. Weger, U. Langsenlehner, P. Krippl, H. Kerl, H. Samonigg, and J. Smolle, "The use of confocal 
laser-scanning microscopy in microsurgery for invasive squamous cell carcinoma," Br. J. Dermatol. 156, 81-84 (2007).

7. W. Denk, J. H. Strickler, and W. W. Webb, "Two-photon laser scanning fluorescence microscopy," Science 248, 73-76 (1990).

8. K. Konig and I. Riemann, "High-resolution multiphoton tomography of human skin with subcellular spatial resolution and picosecond time resolution," J. Biomed. Opt. 8, 432-439 (2003).

9. S.-J. Lin, S.-H. Jee, and C.-Y. Dong, "Multiphoton microscopy: a new paradigm in dermatological imaging," Eur. J. Dermatol. 17, 361-366 (2007).

10. P. T. So, C. Y. Dong, B. R. Masters, and K. M. Berland, "Two-photon excitation fluorescence microscopy," Аnпи. Rev. Biomed. Eng. 2 399-429 (2000).

11. W. R. Zipfel, R. M. Williams, R. Christie, A. Y. Nikitin, B. T. Hyman, and W. W. Webb, "Live tissue intrinsic emission microscopy using multiphoton-excited native fluorescence and second harmonic generation," Proc. Natl. Acad. Sci. U.S.A. 100, 7075-7080 (2003).

12. P. J. Campagnola and L. M. Loew, "Second-harmonic imaging microscopy for visualizing biomolecular arrays in cells, tissues and organisms," Nat. Biotechnol. 21, 1356-1360 (2003).

13. A. T. Yeh, B. Choi, J. S. Nelson, and B. J. Tromberg, "Reversible dissociation of collagen in tissues," J. Invest. Dermatol. 121, 1332$1335(2003)$

14. Y. Sun, W.-L. Chen, S.-J. Lin, S.-H. Jee, Y.-F. Chen, L.-C. Lin, P. T. C. So, and C.-Y. Dong, "Investigating mechanisms of collagen thermal denaturation by high resolution second-harmonic generation imaging," Biophys. J. 91, 2620-2625 (2006).

15. S.-J. Lin, R.-. Wu, Jr., H.-Y. Tan, W. Lo, W.-C. Lin, T.-H. Young, C.-J. Hsu, J.-S. Chen, S.-H. Jee, and C.-Y. Dong, "Evaluating cutaneous photoaging by use of multiphoton fluorescence and secondharmonic generation microscopy," Opt. Lett. 30, 2275-2277 (2005).

16. S.-J. Lin, S.-H. Jee, C.-J. Kuo, R. Wu, Jr., W.-C. Lin, J.-S. Chen, Y.-H. Liao, C.-J. Hsu, T.-F. Tsai, Y.-F. Chen, and C.-Y. Dong, "Discrimination of basal cell carcinoma from normal dermal stroma by quantitative multiphoton imaging," Opt. Lett. 31, 2756-2758 (2006).

17. B.-G. Wang, I. Riemann, H. Schubert, D. Schweitzer, K. Konig, and K.-J. Halbhuber, "Multiphoton microscopy for monitoring intratissue femtosecond laser surgery effects," Lasers Surg. Med. 39, 527-533 (2007).

18. T. S. Alster and J. R. Lupton, "Erbium:YAG cutaneous laser resurfacing," Dermatol. Clin. 19, 453-466 (2001).

19. W.-R. Lee, S.-C. Shen, C.-R. Liu, C.-L. Fang, C.-H. Hu, and J.-Y. Fang, "Erbium:YAG laser-mediated oligonucleotide and DNA delivery via the skin: an animal study," J. Controlled Release 115, 344353 (2006).

20. M.-G. Lin, T.-L. Yang, C.-T. Chiang, H.-C. Kao, J.-N. Lee, W. Lo, S.-H. Jee, Y.-F. Chen, C.-Y. Dong, and S.-J. Lin, "Evaluation of dermal thermal damage by multiphoton autofluorescence and secondharmonic-generation microscopy," J. Biomed. Opt. 11, 064006 (2006).

21. D. C. Swartzendruber, A. Manganaro, K. C. Madison, M. Kremer, P. W. Wertz, and C. A. Squier, "Organization of the intercellular spaces of porcine epidermal and palatal stratum corneum: a quantitative study employing ruthenium tetroxide," Cell Tissue Res. 279, 271-276 (1995).

22. D. C. Swartzendruber, I. H. Burnett, P. W. Wertz, K. C. Madison, and C. A. Squier, "Osmium tetroxide and ruthenium tetroxide are complementary reagents for the preparation of epidermal samples for transmission electron microscopy," J. Invest. Dermatol. 104, 417420 (1995).

23. K. C. Madison, "Barrier function of the skin: 'la raison d'etre' of the epidermis," J. Invest. Dermatol. 121, 231-241 (2003).

24. S. J. Lin, W. Lo, H. Y. Tan, J. Y. Chan, W. L. Chen, S. H. Wang, Y. Sun, W. C. Lin, J. S. Chen, C. J. Hsu, J. W. Tjiu, H. S. Yu, S. H. Jee, and C. Y. Dong, "Prediction of heat-induced collagen shrinkage by use of second harmonic generation microscopy," J. Biomed. Opt. 11, 34020 (2006).

25. E. V. Ross, Y. Domankevitz, M. Skrobal, and R. R. Anderson, "Effects of $\mathrm{CO} 2$ laser pulse duration in ablation and residual thermal damage: implications for skin resurfacing," Lasers Surg. Med. 19, 123-129 (1996).

26. S. Thomsen, "Pathologic analysis of photothermal and photome- chanical effects of laser-tissue interactions," Photochem. Photobiol. 53, 825-835 (1991).

27. E. V. Ross, G. S. Naseef, J. R. McKinlay, D. J. Barnette, M. Skrobal, J. Grevelink, and R. R. Anderson, "Comparison of carbon dioxide laser, erbium:YAG laser, dermabrasion, and dermatome: a study of thermal damage, wound contraction, and wound healing in a live pig model: implications for skin resurfacing," J. Am. Acad. Dermatol. 42 , 92-105 (2000).

28. T. Kuo, M. T. Speyer, W. R. Ries, and L. Reinisch, "Collagen thermal damage and collagen synthesis after cutaneous laser resurfacing," Lasers Surg. Med. 23, 66-71 (1998).

29. S.-J. Lin, C.-Y. Hsiao, Y. Sun, W. Lo, W.-C. Lin, G.-J. Jan, S.-H. Jee, and C.-Y. Dong, "Monitoring the thermally induced structural transitions of collagen by use of second-harmonic generation microscopy," Opt. Lett. 30, 622-624 (2005).

30. W.-R. Lee, S.-C. Shen, W. Kuo-Hsien, C.-H. Hu, and J.-Y. Fang, "Lasers and microdermabrasion enhance and control topical delivery of vitamin C," J. Invest. Dermatol. 121, 1118-1125 (2003).

31. J. Y. Fang, W. R. Lee, S. C. Shen, Y. P. Fang, and C. H. Hu, "Enhancement of topical 5-aminolaevulinic acid delivery by erbium:YAG laser and microdermabrasion: a comparison with iontophoresis and electroporation," Br. J. Dermatol. 151, 132-140 (2004).

32. S.-C. Shen, W.-R. Lee, Y.-P. Fang, C.-H. Hu, and J.-Y. Fang, "In vitro percutaneous absorption and in vivo protoporphyrin IX accumulation in skin and tumors after topical 5-aminolevulinic acid application with enhancement using an erbium:YAG laser," J. Pharm. Sci. 95 , 929-938 (2006).

33. J. N. Lee, S. H. Jee, C. C. Chan, W. Lo, C. Y. Dong, and S. J. Lin, "The effects of depilatory agents as penetration enhancers on human stratum corneum structures," J. Invest. Dermatol. 128, 2240-7 (2008).

34. N. Kanikkannan, J. Singh, and P. Ramarao, "Transdermal iontophoretic delivery of bovine insulin and monomeric human insulin analogue," J. Controlled Release 59, 99-105 (1999).

35. C. A. Zakzewski, J. Wasilewski, P. Cawley, and W. Ford, "Transdermal delivery of regular insulin to chronic diabetic rats: effect of skin preparation and electrical enhancement," J. Controlled Release 50, 267-272 (1998).

36. B. Yu, K. H. Kim, P. T. C. So, D. Blankschtein, and R. Langer, "Visualization of oleic acid-induced transdermal diffusion pathways using two-photon fluorescence microscopy," J. Invest. Dermatol. 120, 448-455 (2003).

37. Y. Sun, W. Lo, S.-J. Lin, S.-H. Jee, and C.-Y. Dong, "Multiphoton polarization and generalized polarization microscopy reveal oleicacid-induced structural changes in intercellular lipid layers of the skin," Opt. Lett. 29, 2013-2015 (2004).

38. F. Stracke, B. Weiss, C.-M. Lehr, K. Konig, U. F. Schaefer, and M. Schneider, "Multiphoton microscopy for the investigation of dermal penetration of nanoparticle-borne drugs," J. Invest. Dermatol. 126 2224-2233 (2006).

39. M. H. Niemz, Laser-Tissue Interactions: Fundamentals and Applications, Springer-Verlag, New York (2004).

40. B. Majaron, S. M. Srinivas, H. E. Huang, and J. S. Nelson, "Deep coagulation of dermal collagen with repetitive Er:YAG laser irradiation," Lasers Surg. Med. 26, 215-222 (2000).

41. M. J. van Gemert, D. J. Smithies, W. Verkruysse, T. E. Milner, and J. S. Nelson, "Wavelengths for port wine stain laser treatment: influence of vessel radius and skin anatomy," Phys. Med. Biol. 42, 41-50 (1997).

42. J. C. Malone, A. F. Hood, T. Conley, J. Nurnberger, L. A. Baldridge, J. L. Clendenon, K. W. Dunn, and C. L. Phillips, "Three-dimensional imaging of human skin and mucosa by two-photon laser scanning microscopy," J. Cutan Pathol. 29, 453-458 (2002).

43. B. Choi, W. Jia, J. Channual, K. M. Kelly, and J. Lotfi, "The importance of long-term monitoring to evaluate the microvascular response to light-based therapies," J. Invest. Dermatol. 128, 485-488 (2008).

44. M. J. Koehler, K. Konig, P. Elsner, R. Buckle, and M. Kaatz, "In vivo assessment of human skin aging by multiphoton laser scanning tomography," Opt. Lett. 31, 2879-2881 (2006).

45. K. Konig, A. Ehlers, F. Stracke, and I. Riemann, "In vivo drug screening in human skin using femtosecond laser multiphoton tomography," Skin Pharmacol. Physiol. 19, 78-88 (2006). 